See discussions, stats, and author profiles for this publication at: https://www.researchgate.net/publication/327915850

\title{
More Attention and less repetitive behaviors using a robot with children with autism
}

Conference Paper · August 2018

DOI: 10.1109/ROMAN.2018.8525747

CITATIONS

0

6 authors, including:

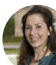

Andreia Costa

University of Luxembourg

11 PUBLICATIONS 15 CITATIONS

SEE PROFILE

Pouyan Ziafati

(3) University of Luxembourg

8 PUBLICATIONS 18 CITATIONS

SEE PROFILE

Some of the authors of this publication are also working on these related projects:

Project RationalArchitecture View project

Project CIFIL: Characterization of Railway Infrastructure using Lidar View project
READS

115

Charpiot Louise

Université Charles-de-Gaulle Lille 3

3 PUBLICATIONS 1 CITATION

SEE PROFILE 


\title{
More Attention and Less Repetitive and Stereotyped Behaviors using a Robot with Children with Autism
}

\author{
Andreia P. Costa, Louise Charpiot, Francisco Rodríguez Lera, Pouyan Ziafati ${ }^{1}$, Aida Nazarikhorram ${ }^{1}$, \\ Leendert van der Torre, and Georges Steffgen, University of Luxembourg
}

\begin{abstract}
The aim of the present study was to assess the usefulness of QTrobot, a socially assistive robot, in interventions with children with autism spectrum disorder (ASD) by assessing children's attention, imitation, and presence of repetitive and stereotyped behaviors. Fifteen children diagnosed with ASD, aged from 4 to 14 years participated in two short interactions, one with a person and one with the robot. Statistical analyses revealed that children directed more attention towards the robot than towards the person, imitated the robot as much as the person, and engaged in fewer repetitive or stereotyped behaviors with the robot than with the person. These results support previous research demonstrating the usefulness of robots in short interactions with children with ASD and provide new evidence to the usefulness of robots in reducing repetitive and stereotyped behaviors in children with ASD, which can affect children's learning.
\end{abstract}

\section{INTRODUCTION}

Autism spectrum disorder (ASD) is a neurodevelopmental disorder characterized by significant difficulties in communication, social interaction, as well as restricted and repetitive behaviors and interests [1]. ASD is present in approximately $1 \%$ of the population [2] and the impact of the deficits can range from mild to severe and impair social, occupational, and functional domains.

People with ASD have a higher prevalence of mental health issues than their neurotypical counterparts and people with other disabilities [3, 4]. Anger outbursts, self-injurious behaviors, anxiety, and depression are among the main reasons why parents of children with ASD seek professional help [5]. These mental health issues have repercussions into social competence, peer acceptance, adaptive development, and increase the risk of psychiatric diagnoses in adulthood $[6,7]$. Therefore, teaching social and emotional abilities to children with ASD is fundamental to foster their well-being and the well-being of their families. However, interventions for children with ASD are costly [8] and the services offered are often insufficient or inadequate [9]. To deal with these challenges, the services provided to children with ASD must be made more efficient and the quality should be improved.

\footnotetext{
*Research supported by Luxembourg National Research Fund. Corresponding author: Andreia P. Costa; phone: 003524666449693 ; e-mail: andreia.pintocosta@uni.lu
}

${ }^{1}$ Pouyan Ziafati and Aida Nazarikhorram are founders of LuxAI S.A., a social robotic spinoff from the University of Luxembourg. In order to avoid any conflict of interest, they do not participate in parts of this research related to scripting, conducting, testing, analysing, and reporting QTrobot applications and experiments for children with ASD.
Robot-assisted therapy has been proven in the past years to be useful for children with ASD [10]. Socially assistive robots can be beneficial for individuals with ASD because they are rule-based and predictable systems that can repeat patterns and can be organized and understood in a systematic way. This corresponds to the learning characteristics of children with ASD, who have a desire for sameness and repetition as well as an interest in inanimate objects [1].

Additionally, and in contrast to other technologies (e.g. computer software, tablet applications, and virtual environments), interactive physical robots provide embodied multi-modal aspects that are important for interpersonal relations [11]. These characteristics can make interactions with robots more compelling to children with ASD than interactions with a human therapist [11, 12]. Additionally, robots provide novel sensory stimuli [13], which can stimulate children's interest and increase assimilation of content. Therefore, studying the interaction of children with ASD with robots provides us with valuable information as to whether robots can engage children and be useful teachers to deliver interventions.

Previous research has shown the effectiveness of robots in increasing attention [14], joint attention [15], cooperation [16], imitation [17], and communication [18] in people with ASD in comparison to a baseline. However, results regarding whether robots can be at least as effective as people to teach abilities to children with ASD are mixed. Some studies have found that robots are better than humans at eliciting attention [19, 20], joint attention [21], communication [22], and imitation [19, 23]. But other studies have found that a robot was as good as a person at eliciting communication [24], joint attention, and motor initiation [25] and that a robot was worse than a person at eliciting imitation of body movements [21] and joint attention [26].

Additionally, a crucial aspect of the behavior of children with ASD has been missing from previous research on the robots' efficacy with children with ASD - the impact of robots in their repetitive and stereotyped behaviors. Repetitive and stereotyped behaviors are frequent among children with ASD and are thought to induce self-stimulation by creating overarousal [27] or to act as soothing behaviors by providing an escape from an over-stimulating environment or disturbing inputs [28]. Repetitive and stereotyped behaviors can interfere with learning opportunities [29] and lead to overselective attention and to difficulties in shifting attention [27], which are fundamental in the learning process. 
In the only study that we found reporting on the impact of robots in the repetitive and stereotyped behaviors of children with ASD, it was found that the four children interacting with the robot engaged in fewer repetitive behaviors with their favorite toy and had no repetitive or stereotyped behavior toward the robot [21]. On the one hand, robots are predictable, repetitive systems that can be understood by children with ASD in a systematic way. On the other hand, many children with ASD feel fascinated and excited by meeting a robot. For these reasons, interacting with a robot can provide an arousing experience to a child with ASD while at the same time being less disturbing than an interaction with a person.

In the present study, we aim to test whether QTrobot, a socially assistive robot, can be useful in interventions with children with ASD by assessing whether children's attention and imitation is as good with the robot as with a person. More importantly, we want to assess whether, children's repetitive or stereotyped behaviors are lower in the presence of QTrobot than in the presence of a person.

\section{II.METHODS}

\section{A. Participants}

A convenience sample of 15 children previously diagnosed with ASD (all boys), aged 4 to 14 years $(M=$ 9.73; $S D=3.38$ ), accompanied by at least one of their parents participated in this study. Children's characteristics are described in Table 1.

Participation was open to all children diagnosed with ASD aged from 4 to 16 years. The diagnosis had to be established by an expert in the light of an assessment based on DSM criteria [1] and recognized by the country's health authorities. Children's autism-related social impairments were assessed through the Social Responsiveness Scale 2 (SRS-2; [30]) and IQ was assessed with the Wechsler Nonverbal Scale of Ability (WNV; [31]). Participants were part of a larger study on the use of a robot to teach emotional abilities to children with ASD.

TABLE I. Children's characteristics on their age, ASD severity, measured non-verbal IQ, and communication ability (verbal or non-verbal)

\begin{tabular}{|c|c|c|c|c|}
\hline \# & Age & $\begin{array}{l}\text { ASD } \\
\text { Severity }^{\text {a }}\end{array}$ & $\mathbf{I Q}^{\mathbf{b}}$ & $\begin{array}{l}\text { Verbal }(\mathrm{V}) \\
\text { or non- } \\
\text { verbal }(\mathrm{NV})\end{array}$ \\
\hline 1 & 13.67 & Severe & $80-120$ & $\mathrm{~V}$ \\
\hline 2 & 8.19 & Severe & $<80$ & V \\
\hline 3 & 13.49 & Severe & $>120$ & V \\
\hline 4 & 4.14 & Severe & $<80$ & NV \\
\hline 5 & 4.54 & Mild & $<80$ & NV \\
\hline 6 & 11.48 & Moderate & $<80$ & NV \\
\hline 7 & 8.85 & Severe & $80-120$ & V \\
\hline 8 & 9.22 & Moderate & $80-120$ & V \\
\hline 9 & 8.21 & Severe & $<80$ & V \\
\hline 10 & 14.46 & Severe & $80-120$ & V \\
\hline 11 & 14.48 & Moderate & $<80$ & NV \\
\hline 12 & 8.22 & Severe & $80-120$ & V \\
\hline 13 & 9.58 & Severe & $80-120$ & V \\
\hline 14 & 6.04 & Mild & $<80$ & V \\
\hline 15 & 11.38 & Severe & $<80$ & V \\
\hline
\end{tabular}

\section{B. Material}

\section{1) Robot}

The robot utilized, QTrobot (see Fig. 1), is a commercially available (LuxAI S.A.) child-sized plastic bodied humanoid robot (about $63 \mathrm{~cm}$ tall and $5 \mathrm{~kg}$ ) used in other recent applications for children with ASD [32]. QTrobot has an expressive social appearance and its screen allows the presentation of animated faces. It has 12 degrees of freedom to present upper-body gestures. Eight degrees of freedom are motor-controlled, two in each shoulder, one in each arm plus pitch and yaw movements of the head. The other four degrees of freedom, one in each wrist and one in each hand, are manually configured. QTrobot has a RealSense 3D camera mounted on its forehead and is provided with a microphone array. QTrobot is powered with an Intel NUC processor and Ubuntu 16.04 Lts, and provides a native ROS interface to program it in Python or $\mathrm{C}++$ programming languages. QTrobot also provides a visual programming interface for IT non-experts, used in this study, to easily script custom applications and control the robot by an Android application from tablets and smart phones. In the present study, the robot's interactions with the children were pre-scripted and controlled by the experimenter via a tablet.

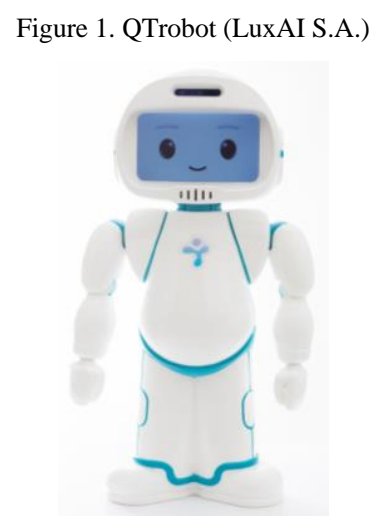

\section{2) Interview}

To compare children's interaction with the robot and with a human, we have created two interviews similar in structure but with different items ( $\mathrm{A}$ and $\mathrm{B}$; see appendices $\mathrm{A}$ and $\mathrm{B}$ ). During each interview, the child was sitting at a desk facing the interview partner (person or robot; see Fig. 2). Each interview started with the interview partner asking the child his name. Then, the person or the robot presented themselves and asked three questions to the child about his preferences (e.g. favorite animal, favorite color, etc.). Then the interview partner told a short story and asked the child whether he liked the story. To finish, the interview partner asked the child to do an imitation game involving four gestures with the arms (e.g. left arm up, right arm up, left arm to the side, right arm to the side). Interviews lasted between $1.5 \mathrm{~min}$ to $4.3 \mathrm{~min}$ (person: $M=2.2 ; S D=0.48$; robot: $M=3.2 ; S D=0.58$ ). The interview partners were two females. During the interaction with the person, the person acted naturally with the child as in a typical adult-child interaction (i.e. meeting child's gazes, 
smiling at the child, etc.). During interactions with the person and robot sufficient time was allowed for the child to answer.

Figure 2. Experiment setup with person (left) and with robot (right)

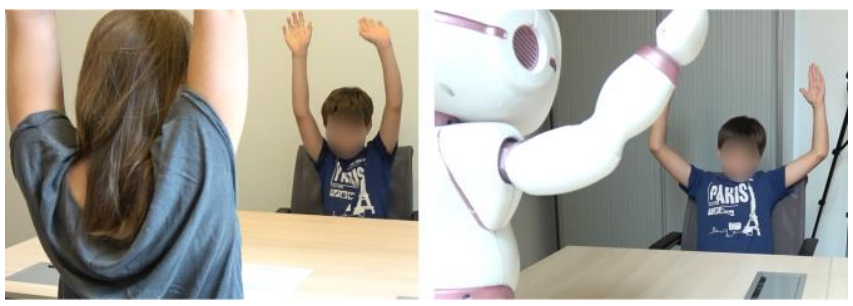

\section{Procedure}

Parents and children were invited to participate in the study through a letter distributed by institutions for children with ASD in Luxembourg. The study was reviewed and approved by the University of Luxembourg's ethics review panel (approval number: ERP17-017-SAR-A) and parents read and signed informed consent forms for participation and data collection.

The study took place in one 2-hours long visit. During the visit, parents were requested to fill out questionnaires concerning their children's emotional ability, internalizing and externalizing problems, and autism-related social impairments. During that time, children were first invited to a room where a person did Interview A with the child (see Appendix A). After that, children's IQ was assessed as well as children's emotional ability in different domains (recognition and differentiation of emotions, emotion regulation strategies during scenarios, and emotion regulation ability during a frustration-eliciting situation). At the end, children were invited to another room where QTrobot did Interview B with the child (see Appendix B). The order of having the interview with the person first and with the robot at last was chosen for two reasons. First, to reduce the novelty difference between the person and the robot (children had never met the robot before), we wanted the child to see the person doing the interview for the first time during the interview. Second, many children, after meeting with the robot, wanted to stay with the robot and it was difficult to engage them in the other necessary measures afterwards due to repeated questions about the robot or persistent enquiries about when they would see the robot again.

\section{Analysis}

The videos of the interviews were annotated by two independent observers in which one of the observers was not aware of the study's objectives. For each child, observers annotated both the child's interview with the person and with the robot. To assess children's attention to the interview partner, the number of children's gazes towards the interview partner and the duration of each gaze were annotated. To assess children's imitation of the interview partner's actions, the number of imitations (max. 4 imitations) was annotated. Finally, to assess children's repetitive and stereotyped behaviors, the number of chains of repetitive and stereotyped behaviors was counted as well as the number of repetitions per chain. A chain of repetitive and stereotyped behaviors was defined as an uninterrupted sequence of the same type of repetitive and stereotyped behavior. If the child paused the behavior to engage in a different behavior, it was counted as one chain. If the child, after the pause, engaged again in the same or in a different repetitive and stereotyped behavior, it was counted as a second chain. A behavior was considered repetitive and stereotyped if the same behavior occurred at least 3 consecutive times (e.g. hand tapping on the table, rocking back and forth)

\section{RESULTS}

The non-parametric Wilcoxon signed-rank test was used to compare children's attention, imitation, and presence of repetitive and stereotyped behaviors during the interview with the person and with the robot.

Regarding children's attention we found that on average children had more gazes towards the person $(M=11.02 ; S D$ $=6.63)$ than towards the robot $(M=8.79 ; S D=5.16)$ but this difference was not statistically significant, $T=52, p=.454, r$ $=.09$ (see Fig. 3, Panel A). However, children's average duration per gaze was significantly lower for the gazes directed at the person $(M=2.73 ; S D=2.74)$ than those directed at the robot $(M=6.23 ; S D=6.88), T=17, p=.046$, $r=.39$ (see Fig. 3, Panel B). Overall, children spent a significantly lower percentage of time looking at the person $(M=41.28 ; S D=26.83)$ than at the $\operatorname{robot}(M=68.21 ; S D=$ 19.78), $T=10, p=.013, r=.49$ (see Fig. 3, Panel C).

Regarding children's imitation of the interview partner's actions, we found that on average children imitated more often the person $(M=3.85 ; S D=0.55)$ than the $\operatorname{robot}(M=$ 2.93; $S D=1.83$ ) but this difference was not statistically significant, $T=3, p=.180, r=.26$ (see Fig. 3, Panel D).

Figure 3. Panel A: number of gazes per minute; Panel B: gaze average duration (in seconds); Panel C: percentage of gaze duration; Panel D: number of imitations. Error bars represent standard deviations.

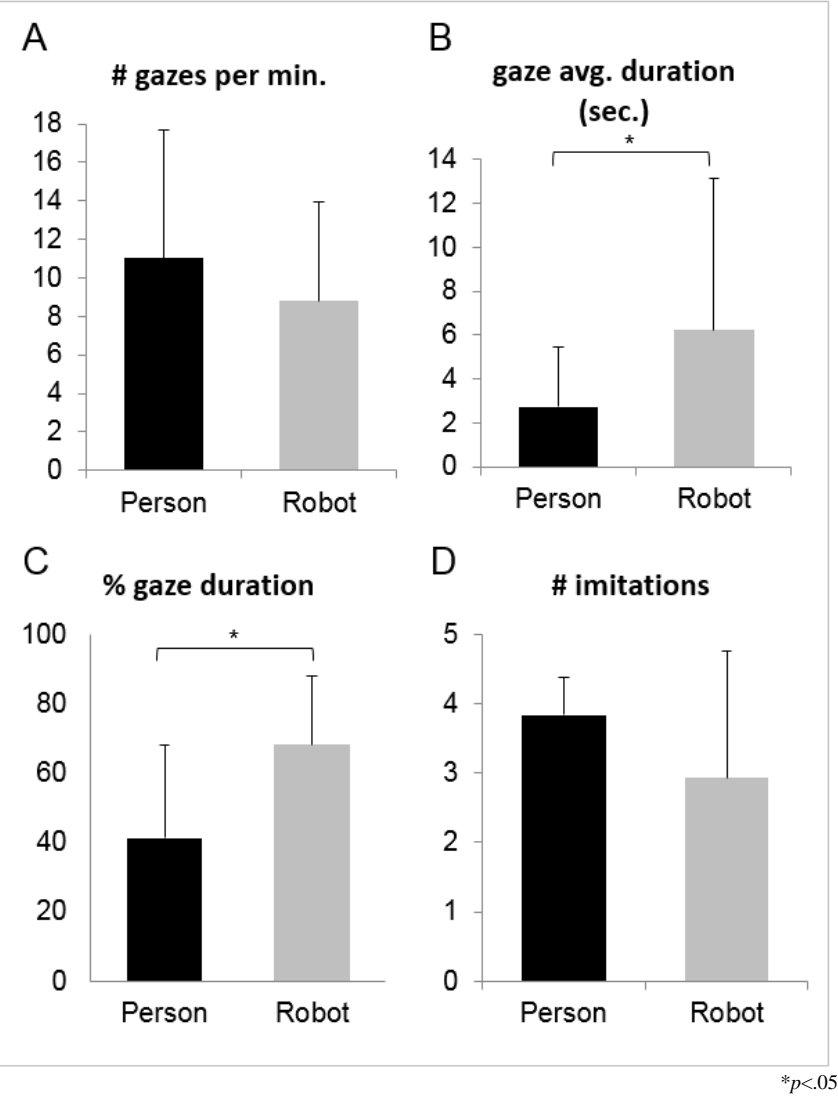


Regarding the presence of repetitive and stereotyped behaviors, we found that children had significantly more chains of repetitive and stereotyped behaviors during the interaction with the person $(M=3.31 ; S D=4.16)$ than in the interaction with the robot $(M=1.05 ; S D=1.91), T=48, p=$ $.037, r=.38$ (see Fig. 4, Panel A). Additionally, when children engaged in chains of repetitive and stereotyped behaviors, those were significantly more frequent per minute in the interaction with the person $(M=13.56 ; S D=17.68)$ than in the interaction with the robot $(M=4.45 ; S D=9.40)$, $T=40, p=.0 .38, r=.39$ (see Fig. 4, Panel B).

Figure 4. Panel A: number of chains of repetitive and stereotyped behaviors per minute; Panel B: number of repetitive and stereotyped behaviors within chains per minute. Error bars represent standard deviations.

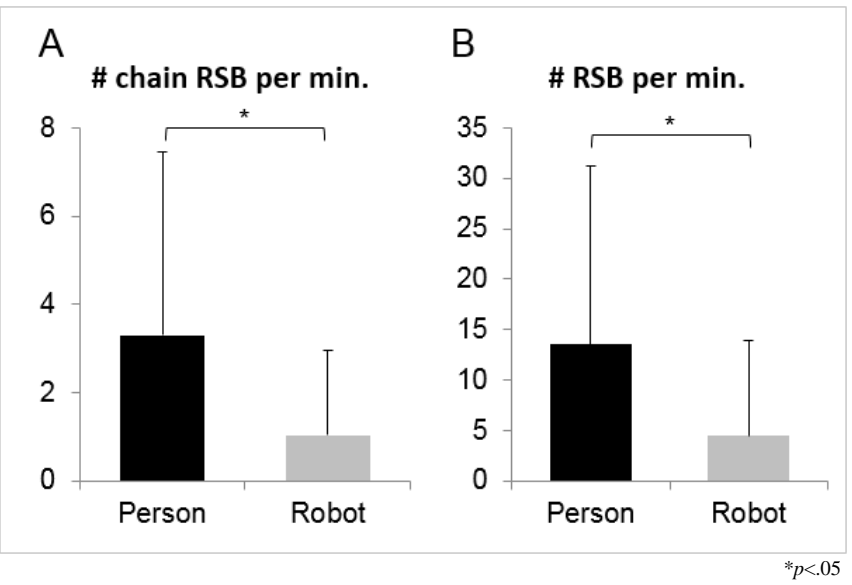

Since children's age varied from 4 to 14 years, Pearson correlational analyses were conducted to assess the relation between children's age and their gaze, imitation, and repetitive and stereotyped behaviors during the interactions with the person and the robot. Results revealed that age was not significantly associated with any of the measures during the person interaction nor during the robot interaction.

\section{DiscuSSION AND CONCLUSIONS}

The aim of the present study was to assess whether QTrobot can be useful in interventions with children with ASD. For that, we compared 15 children with ASD in a short interaction with a person and with the robot. We assessed children's gazes towards the interview partner as indications of children's attention, as well as children's imitations of the interview partner, and the presence of repetitive and stereotyped behaviors during the interaction.

In terms of attention, we found that, even though the difference was not statistically significant, children directed their gaze more often towards the person than towards the robot. This indicates that children divert their gaze as often from the person as from the robot. However, when children looked at the interaction partner, they looked significantly longer at the robot than at the person. This can indicate that children are more comfortable looking at the robot than looking at the person. This is also confirmed by the fact that, overall, during the entire interaction, children significantly spent a greater proportion of time looking at the robot than looking at the person. These results are in agreement with the few studies that compared children's attention towards a robot and towards a person and found that children with ASD directed more attention towards the robot $[19,20]$.

In terms of children's imitation of the interview partner, we found that children imitated on average more often the person than the robot but the difference was not statistically significant. Therefore, we can conclude that children with ASD imitate as much QTrobot as a person. Our results are therefore not in line neither with studies that found that children with ASD imitate more a robot than a person [19, 23], nor with those that found that children with ASD imitated more a person than a robot [21].

Finally, an important aspect to evaluate the efficacy of a robot to be used in interventions with children with ASD is the presence of repetitive or stereotyped behaviors during the interaction. Repetitive or stereotyped behaviors can have detrimental effects in children's learning [29] and attention [27] and therefore, fewer of these behaviors could enable more opportunities for the child to engage with the teaching partner and learn. However, this aspect has to date only been assessed in one study with four participants in which they found that children had fewer repetitive or stereotyped behaviors with the robot than with a person [21]. In our study, we found that when children were with the robot they engaged in significantly fewer repetitive or stereotyped behaviors and that when they did, the frequency of the repeated behavior was significantly lower than with a person.

In summary, the present results demonstrate that QTrobot is an engaging robot that can be beneficial to be used with children with ASD. The fact that children direct more attention towards the robot, imitate the robot as much as a person, and engage in fewer repetitive or stereotyped behaviors with the robot than with a person represent increased learning opportunities for children with ASD.

It is worth mentioning that the present results can however be due to the brief exposure of children to the robot. It is possible that the robot represents a novelty, which triggers heightened attention and thus reduces concomitant repetitive and stereotyped behaviors and that this effect could disappear over time, enabling us to only conclude about the present effects for short periods of interaction. Studies with an evaluation of children's interaction with a robot for longer periods of time are needed to ascertain the long-term benefits of using a robot with children with ASD.

Additionally, because the order of exposure to each interview partner was not counterbalanced across participants and interview conditions, it is possible that some effects may be due to the order of exposure and to the moment when children were exposed to the robot (after two hours of evaluations). However, attention tends to drop with tiredness. Similarly, irritability and repetitive and stereotyped behaviors are more frequent in children with ASD when they are tired, such as when they are sleep deprived [33]. Therefore, if the order of exposure would affect the interactions, it would be expected that it would affect the interaction with the robot in a way that attention would be decreased and repetitive and stereotyped behaviors would increase compared to the earlier interaction with the person. Even more so because the type of interaction with the robot was similar to the one already experienced with the person. However, this was not the case. 
Therefore, the present results provide us with confidence to conclude that the robot was better than the person at eliciting increased attention and decreased repetitive and stereotyped behaviors during a short interaction.

\section{APPENDICES}

Appendix A - Interview A (with person)

\section{Interview A}

Hello! What's your Name?

$$
\text { [wait for child's answer] }
$$

I am [first name]. I am a researcher and I am a specialist on emotions. You know, I'm a little curious and would like to ask you some questions. What's your favourite colour?

What's your favourite game?

$$
\text { wait for child's answer] }
$$

What's your favourite fruit?

[wait for child's answer]

I am going to tell you a story now

Once upon a time there was a very hungry wolf. He went to look for food everywhere, but he didn't find anything. Finally, he found a large piece of bread with meat in the hole of a tree. The wolf got into the hole and ate everything. But the food belonged to the woodcutter, who had left but was now returning for his lunchbreak. Instead of his lunch he found the wolf in the hole. When the wolf saw the woodcutter, he wanted to flee. But having eaten all the food he had become too large to get out of the hole and the woodcutter punished him. And that's how the story ends! Did you like my story?

[wait for child's answer]

Now we are going to play an imitation game. I am going to do something, and you are going to do the same. Let's go.

One arm up.

Then the other.

[left arm up - wait for child's imitation]

Then one arm to the side.

[right arm up - wait for child's imitation]

[left arm open to the left - wait for child's imitation]

And the other one, too.

Thank you!
Appendix B - Interview B (with robot)

\section{Interview B}

Hello! What's your Name?

[wait for child's answer]

I am [first name]. I am a researcher and I am a specialist on emotions.

You know, I'm a little curious and would like to ask you some questions.

What's your favourite animal?

[wait for child's answer]

What's your favourite cartoon movie?
[wait for child's answer]

What's your favourite dessert?

[wait for child's answer]

I am going to tell you a story now.

Once upon a time, there was a big lion who lived in the forest. One day after his lunch he fell asleep under a tree. A little mouse came and played on top of the lion. Suddenly the lion woke up angrily. He saw the mouse shaking with fear. The lion wanted to eat the mouse, but the mouse asked him for forgiveness. The lion had sympathy for the mouse and let it run away. Some days later the lion got caught in a hunter's net. Then the little mouse came and chewed through the net, so the lion could escape. After this the lion and the mouse became very good friends. And that's how the story ends!

Did you like my story? [wait for child's answer]

Now we are going to play an imitation game. I am going to do something, and you are going to do the same. Let's go.

One arm to the side.

Then the other.

[left arm open to the left - wait for child's imitation]

[right arm open to the right - wait for child's imitation]

Then one arm up.

And the other one too.

left arm up - wait for child's imitation]

Thank you! [right arm up - wait for child's imitation]

\section{REFERENCES}

[1] American Psychiatric Association 2013. Diagnostic and statistical manual of mental disorders (5th ed.) Washington, DC: Author.

[2] Elsabbagh M., Divan G., Yun-Joo, K., et al. (2012). Global prevalence of autism and other pervasive developmental disorders. Aut. Res., 5, 160-179.

[3] Bauminger, N., Solomon, M., \& Rogers, S. (2010). Externalizing and internalizing behaviors in ASD. Aut. Res., 3(3), 101-112.

[4] Brereton, A. V., Tonge, B. J., \& Einfeld, S. L. (2006). Psychopathology in children and adolescents with autism compared to young people with intellectual disability. JADD, 36(7), 863-870.

[5] Samson A.C. \& Tornare E. (2015). Perturbations émotionnelles et leurs remédiations dans le trouble du spectre de l'autisme. ANAE, 139, 001009.

[6] Burt K.B., Obradović J., Long J.D., \& Masten A.S. (2008). The interplay of social competence and psychopathology over 20 years: Testing transactional and cascade models. Child Dev., 79(2), 359-374.

[7] Cicchetti D. \& Toth S.L., editors (1991). Internalizing and externalizing expressions of dysfunction. Erlbaum; Hillsdale, NJ.

[8] Buescher A.V., Cidav Z., Knapp M., \& Mandell S. (2014). Costs of autism spectrum disorders in the UK and the US. JAMA Pediatrics, $168,721-728$.

[9] Gustavsson, A., Svensson, M., Jacobi, F., Allgulander, et al (2011). Cost of disorders of the brain in Europe 2010. Eur. Neur., 21(10), 718779.

[10] Thill, S., Pop, C. A., Belpaeme, T., Ziemke, T., \& Vanderborght, B. (2012). Robot-assisted therapy for autism spectrum disorders with (partially) autonomous control: Challenges and outlook. Paladyn, 3(4), 209-217.

[11] Dautenhahn K. \& Werry I. (2004). Towards interactive robots in autism therapy: Background, motivation and challenges. Prag. \& Cog., 12, 1-35.

[12] Robins B., Dautenhahn K., \& Dubowski J. (2006). Does appearance matter in the interaction of children with autism with a humanoid robot? Inter. Studies, 7, 3, 509-512.

[13] Scassellati B., Admoni H., \& Matarić M. (2012). Robots for use in autism research. Annual Review of Biomedical Engineering, 14, 275 294.

[14] Bird G., Leighton J., Press C., \& Heyes C. (2007). Intact automatic imitation of human and robot actions in autism spectrum disorders. Proc.: Bio. Sci., 274, 1628, 3027-3031.

[15] De Silva P.R.S., Tadano K., Saito A., et al. (2009). Therapeuticassisted robot for children with autism. IROS (pp. 3561-3567). NY: ACM Press.

[16] Wainer J., Ferrari E., Dautenhahn K., \& Robins B. (2010). The effectiveness of using a robotics class to foster collaboration among groups of children with autism in an exploratory study. Personal and Ubiquitous Computing 14, 5 (Jul. 2010), 445-455.

[17] Robins B., Dickerson P., Stribling P., \& Dautenhahn K. (2004). Robotmediated joint attention in children with autism: A case study in robothuman interaction. Interaction Studies, 5, 2, 161-198.

[18] Taheri, A. R., Alemi, M., Meghdari, A., PourEtemad, H. R., \& Basiri, N. M. (2014, October). Social robots as assistants for autism therapy in Iran: Research in progress. In Robotics and Mechatronics (ICRoM), 2014 Second RSI/ISM (pp. 760-766).

[19] Warren, Z., Zheng, Z., Das, S., Young, E. M., Swanson, A., Weitlauf, A., \& Sarkar, N. (2015). Brief report: development of a robotic intervention platform for young children with ASD. Journal of autism and developmental disorders, 45(12), 3870-3876.

[20] Bekele, E., Crittendon, J. A., Swanson, A., Sarkar, N., \& Warren, Z. E. (2014). Pilot clinical application of an adaptive robotic system for young children with autism. Autism, 18(5), 598-608.

[21] Duquette, A., Michaud, F., \& Mercier, H. (2008). Exploring the use of a mobile robot as an imitation agent with children with low-functioning autism. Autonomous Robots, 24(2), 147-157.

[22] Vanderborght, B., Simut, R., Saldien, J., Pop, C., Rusu, A. S., Pintea, S., ... \& David, D. O. (2012). Using the social robot probo as a social story telling agent for children with ASD. Interaction Studies, 13(3), 348-372.

[23] Pierno, A. C., Mari, M., Lusher, D., \& Castiello, U. (2008). Robotic movement elicits visuomotor priming in children with autism. 
Neuropsychologia, 46(2), 448-454.

[24] Huskens, B., Palmen, A., Van der Werff, M., Lourens, T., \& Barakova, E. (2015). Improving collaborative play between children with autism spectrum disorders and their siblings: The effectiveness of a robotmediated intervention based on Lego® therapy. Journal of autism and developmental disorders, 45(11), 3746-3755.

[25] Tapus, A., Peca, A., Aly, A., Pop, C., Jisa, L., Pintea, S., ... \& David, D. O. (2012). Children with autism social engagement in interaction with Nao, an imitative robot: A series of single case experiments. Interaction studies, 13(3), 315-347.

[26] Anzalone, S. M., Tilmont, E., Boucenna, S., Xavier, J., Jouen, A. L., Bodeau, N., ... \& Michelangelo Study Group. (2014). How children with autism spectrum disorder behave and explore the 4-dimensional (spatial 3D+ time) environment during a joint attention induction task with a robot. Research in Autism Spectrum Disorders, 8(7), 814-826.

[27] Liss, M., Saulnier, C., Fein, D., \& Kinsbourne, M. (2006). Sensory and attention abnormalities in autistic spectrum disorders. Autism, 10(2), $155-172$.

[28] Gillingham, G. (2000) Autism: A New Understanding. Edmonton, Canada: Tacit

[29] Watt, N., Wetherby, A. M., Barber, A., \& Morgan, L. (2008). Repetitive and stereotyped behaviors in children with autism spectrum disorders in the second year of life. Journal of autism and developmental disorders, 38(8), 1518-1533.

[30] Constantino, J. N., \& Gruber, C. P. (2012). Social Responsiveness Scale-Second Edition (SRS-2). CA: Western Psychological Services

[31] Wechsler, D. \& Naglieri, J.A. (2006). Wechsler nonverbal scale of ability. Person.

[32] Costa A.P., Steffgen G., Lera F., Nazarikhorram A., \& Ziafati P. (2017). Socially assistive robots for teaching emotional abilities to children with ASD. Proceedings 3rd workshop Child-Robot Interaction at HRI.

[33] Schreck, K. A., Mulick, J. A., \& Smith, A. F. (2004). Sleep problems as possible predictors of intensified symptoms of autism. Research in developmental disabilities, 25(1), 57-66. 